Original Paper http://ajol.info/index.php/ijbcs http://indexmedicus.afro.who.int

\title{
Caractérisation des propriétés physico-chimiques et chimiques d'un compost issu d'un mélange de rafles de palmier et de fientes de volaille au Sud-Ouest de la Côte d'Ivoire
}

\author{
Edwige-Gwladys Kawojou GNIMASSOUN ${ }^{1^{*}}$, Jean Baptiste Djétchi ETTIEN ${ }^{1,2}$ et \\ Dominique MASSE ${ }^{3}$
}

\author{
${ }^{I}$ Département des Sciences du Sol, UFR Sciences de la Terre et des Ressources Minières Université Felix \\ Houphouët-Boigny, Cocody-22 BP 582 Abidjan 22, Côte d'Ivoire. \\ ${ }^{2}$ Centre Suisse de Recherches Scientifiques. \\ ${ }^{3}$ UMR ECO\&SOLS (IRD, CIRAD, INRA, SUPAGRO Montpellier, Université de Montpellier). \\ ${ }^{*}$ Corresponding author; E-mail: edwigegnimassoun1@gmail.com; Tel: 0022547854871
}

\section{RESUME}

En Côte d'Ivoire, la pauvreté des sols est une contrainte pour la productivité agricole. L'objectif de cette étude était de produire un compost à base de rafles de palmier afin d'améliorer les propriétés physicochimiques des sols pauvres en matières organiques. Quatre traitements T0 $(720 \mathrm{~kg}$ de rafles $+0 \mathrm{~kg}$ de fientes de volaille), T1 (720 kg de rafles $+200 \mathrm{~kg}$ de fientes de volaille), $\mathrm{T} 2(720 \mathrm{~kg}$ de rafles $+400 \mathrm{~kg}$ de fientes de volaille) et T3 (720 kg de rafles $+600 \mathrm{~kg}$ de fientes de volaille), ont été compostés durant 6 mois. Les teneurs en nutriments et éléments traces métalliques (ETM) dans chacun des traitements ont été évaluées et comparées les unes par rapport aux autres. Le traitement T3 a révélé des teneurs élevées en N (14840 mg. $\left.\mathrm{kg}^{-1}\right), \mathrm{P}(6327,5$ mg.kg $\left.{ }^{-1}\right), \mathrm{K}\left(19930 \mathrm{mg} \cdot \mathrm{kg}^{-1}\right), \mathrm{Mg}\left(14160 \mathrm{mg} \cdot \mathrm{kg}^{-1}\right)$ et $\mathrm{Ca}\left(4140 \mathrm{mg} \cdot \mathrm{kg}^{-1}\right)$ et des concentrations en ETM en deçà des seuils de toxicité avec $\left.\mathrm{Pb}: 88,5 \mathrm{mg} \cdot \mathrm{kg}^{-1}, \mathrm{Zn}: 167,5 \mathrm{mg} \cdot \mathrm{kg}^{-1} \mathrm{et} \mathrm{Cr}: 58 \mathrm{mg} \cdot \mathrm{kg}^{-1}\right)$. Ce compost pourrait donc être utilisé comme amendement pour ces ferralsols marginaux dans le Sud-Ouest de la Côte d'Ivoire.

(C) 2020 International Formulae Group. All rights reserved.

Mots clés : Rafles de palmier, compost, fertilité des sols, ETM, Côte d'Ivoire.

\section{Characterization of the physicochemical and chemical properties of a compost issued from a mixture of palm empty fruit bunch and chicken manure in South West of Côte d'Ivoire}

\begin{abstract}
In Côte d'Ivoire, soil poverty reduces agricultural productivity. The aim of this study is to produce compost based on palm stalks to improve the physico-chemical properties of sandy soils with low organic matter content. Four treatments T0 (720 kg of empty fruit bunch $+0 \mathrm{~kg}$ of chicken manure), T1 (720 kg of empty fruit bunch $+200 \mathrm{~kg}$ of chicken manure), $\mathrm{T} 2$ (720 kg of empty fruit bunch $+400 \mathrm{~kg}$ of chicken manure) and T3 (720 kg of empty fruit bunch $+600 \mathrm{~kg}$ of chicken manure) of mixed various quantities of palm empty fruit bunch and chicken manure were up in field for 6 months. The levels of nutrients and heavy metal contents in each treatment were evaluated and compared against each other. Treatment T3 highlights high levels of N
\end{abstract}


(14840 mg.kg-1), P (6327.5 mg.kg $\left.{ }^{-1}\right), \mathrm{K}$ (19930 mg.kg-1), Mg (14160 mg.kg-1) and Ca (4140 mg.kg-1) and heavy metal content below the toxicity thresholds (Pb: $88.5 \mathrm{mg} \cdot \mathrm{kg}^{-1}, \mathrm{Zn}: 167.5 \mathrm{mg} \cdot \mathrm{kg}^{-1}$ and $\left.\mathrm{Cr}: 58 \mathrm{mg} \cdot \mathrm{kg}^{-1}\right)$. This compost could therefore be used as sandy soil amendment in the South West of Côte d'Ivoire.

(C) 2020 International Formulae Group. All rights reserved.

Keywords: Palm empty fruit bunch, compost, soil fertility, heavy metal, Côte d'Ivoire.

\section{INTRODUCTION}

En milieu tropical, la mise en culture des sols entraîne une diminution rapide du stock de matières organiques et l'apparition de carences en azote $(\mathrm{N})$, phosphore $(\mathrm{P})$, potassium (K) et éléments divers (Yoni et al., 2005). En Côte d'Ivoire, le problème de la baisse de la fertilité des sols est une contrainte pour la productivité agricole (Brou et Chaléart, 2007). Dans les agricultures dites conventionnelles, l'alternative face à cette perte de matière organique et des éléments minéraux est l'usage des engrais chimiques dont le coût excessif ou formules parfois inappropriées est inaccessible aux producteurs (Ettien et al., 2016). L'une des stratégies pour l'enrichissement en matière organique, en particulier les ferralsols, est l'utilisation de tous produits résiduaires organiques sous forme d'amendement ou de fertilisant (Koné et al., 2009). Dans le cas des huileries de palme, les déchets liquides (boues liquides, eau de lavage, etc.) sont rejetés généralement sans traitement dans l'environnement, les cours d'eaux et les déchets solides (condensats, rafles, fibres, coques et amandes cassées, etc) sont en grande partie stockés ou incinérés sans disposition particulière. Cette pratique de gestion inappropriée des déchets de récolte entraine une perte nette de nutriments pour les sols à vocation agricole. De nombreux travaux ont indiqué le fort potentiel des rafles de palmier comme source de nutriments et de matière organique pour améliorer la fertilité des sols (Schuchardt et al., 2002 ; Singh et al., 2010 ; Bessou et al., 2017). La présente étude vise à valoriser les sous- produits de palmier à huile et de façon spécifique à évaluer l'effet des apports croissants de fientes de volaille sur la valeur agronomique des rafles après un processus de compostage. En effet, les rafles étant naturellement fibreuse, l'ajout de fientes de volaille va permettre d'accélérer le processus de leur décomposition. Elles sont très riches en azote et également très disponible du fait de la forte extension des élevages de volailles dans la région étudiée. Le compost a pour objectif de concentrer les teneurs en éléments minéraux du produit résiduaire organique par accélération du processus de minéralisation et l'élévation de la température au cours du compostage permet également d'assainir le produit résiduaire organique des parasites phytopathogène. L'hypothèse est qu'il existe un niveau d'apport optimal de fientes de volaille pour une qualité appropriée d'un compost destiné à fertiliser ou amender des sols sableux des régions forestières humide en Côte d'Ivoire.

\section{MATERIEL ET METHODES \\ Zone d'étude}

L'étude a été réalisée à Grand-Lahou (environ $152 \mathrm{~km}$ d'Abidjan) dans la petite localité N'gorankro 1 avec pour coordonnées géographiques 5०19'59', de latitude Nord et $5^{\circ} 1$ '39", de longitude Ouest (Figure 1). La zone est caractérisée par un climat de type équatorial humide et une pluviométrie variant entre 1500 et $2000 \mathrm{~mm} / \mathrm{an}$. On y distingue 4 saisons alternées : 2 saisons sèches et 2 saisons pluvieuses. La végétation est constituée de forêts dégradées par l'activité humaine. Il existe également des forêts secondaires appartenant au domaine Guinéen. Le site expérimental est un ancien verger de cacao et de palmier à huile. Les sols du site d'étude sont des ferralsols sableux, pauvres en matière organique.

\section{Processus de compostage}

Les rafles de palmier $(\mathrm{R})$ provenant de l'industrie de raffinerie d'huile de la région ont été utilisées comme principal substrat. Ces rafles sont le support fibreux résiduel 
obtenues après égrappage du régime de graine de palme. Elles sont constituées d'environ $60 \%$ de cellulose, 11 à $18 \%$ de lignine et 17 à 22\% d'hémicellulose (Nurhayati et Fauziah, 2013). La fiente de volaille (F) a été collectée dans une ferme de la même région et a été utilisée comme activateur dans le processus de compostage des rafles.

Le mode de compostage choisi lors de cette étude était le compostage aérobie en tas. Sur une surface de $4 \mathrm{~m}^{2}$, un tas de $720 \mathrm{~kg}$ de rafles hachées a été déposé en 6 couches de $120 \mathrm{~kg}$ alternées avec 5 couches de fientes de volaille à différentes doses en fonction des traitements. Chaque traitement était composé de $720 \mathrm{~kg}$ de rafles $+0 \mathrm{~kg}$ de fientes de volaille pour le témoin $\mathrm{T} 0,720 \mathrm{~kg}$ de rafles + $200 \mathrm{~kg}$ de fientes de volaille (soit $120 \mathrm{~kg}$ de rafles $+40 \mathrm{~kg}$ de fientes de volaille par couche) pour T1, $720 \mathrm{~kg}$ de rafles $+400 \mathrm{~kg}$ de fientes de volaille (soit $120 \mathrm{~kg}$ de rafles +80 $\mathrm{kg}$ de fientes de volaille par couche) pour $\mathrm{T} 2$ et $720 \mathrm{~kg}$ de rafles $+600 \mathrm{~kg}$ de fientes de volaille (soit $120 \mathrm{~kg}$ de rafles $+120 \mathrm{~kg}$ de fientes de volaille par couche) pour T3 (Tableau 1). Tous les tas ont été arrosés avec $180 \mathrm{~L}$ d'eau puis recouverts par des feuilles de palme et abrités sous une ombrière afin de conserver l'humidité dans les tas. Les tas ont été retournés à une fréquence bihebdomadaire suivie d'arrosage (lorsque cela était nécessaire) afin de maintenir l'humidité et l'aération. Durant le compostage, tous les 2 jours, la température, l'humidité et le $\mathrm{pH}$ des différents traitements ont été mesurés à l'aide d'un multi paramètre électronique de terrain équipé d'une sonde (AGROS-Thermohygromètre et DSMM60). La quantité d'eau apportée à chaque date de retournement était fonction de l'humidité mesurée dans le tas afin de maintenir cette humidité entre 40 et $60 \%$. L'analyse de l'évolution des paramètres physico-chimiques, couplée à la texture du matériau déterminée par la méthode tactile ont permis d'estimer la maturité des composts. La durée de compostage a été de 181 jours. À la maturité, environ $2 \mathrm{~kg}$ de compost de chaque traitement ont été prélevés et acheminés au laboratoire.

\section{Analyse en laboratoire}

L'humidité massique (Hs) des composts a été déterminée selon la formule suivante :

$$
H_{s}(\%)=\left(\frac{W_{W}-W_{d}}{W_{d}}\right) \times 100
$$

Où $\mathrm{H}_{\mathrm{S}}$ est la teneur en eau en pourcentage du poids de l'échantillon de compost; $\mathrm{W}_{\mathrm{w}}(\mathrm{g})$ représente le poids humide de l'échantillon incluant le poids de l'échantillon de compost et celui de l'eau et $\mathrm{W}_{\mathrm{d}}(\mathrm{g})$ : Poids sec de l'échantillon de compost.

Les mesures du $\mathrm{pH}_{\text {eau }}$ et $\mathrm{du} \mathrm{pH}_{\mathrm{KCl}}$ ont été effectuées sur l'extrait aqueux d'un mélange sol (compost)/solution dans un rapport masse/volume de 1/2,5. Le $\mathrm{pH}$ de chaque extrait aqueux a été mesuré à l'aide d'un pH-mètre Inolab ${ }^{\circledR}$ en triplicate.

L'azote total a été dosé par la méthode Kjedahl. L'extraction du phosphore total a été faite par attaque perchlorique à chaud et son dosage par colorimétrie en présence du vanado-molybdate. Le potassium a été déterminé au spectrophotomètre de flamme. Les concentrations totales en $\mathrm{Ca}, \mathrm{Mg}, \mathrm{Pb}, \mathrm{Zn}$ et $\mathrm{Cr}$ ont été déterminées par spectrométrie d'absorption atomique à flamme.

\section{Minéralisation du carbone et de l'azote}

La minéralisation du carbone et de l'azote a été réalisée au cours d'une incubation des sols dans un bocal hermétique avec le compost T3. Le sol utilisé provient de la parcelle du site expérimental dont les caractéristiques physico-chimiques sont présentées dans le Tableau 2. Pour les incubations, environ $10 \mathrm{~g}$ de sol ont été mélangés à différentes doses de $0 \mathrm{~g}, 0,066 \mathrm{~g}$, $0,133 \mathrm{~g}$ et $0,26 \mathrm{~g}$ du compost $\mathrm{T} 3$ correspondant aux traitements D0, D1, D2 et D3. Ces doses correspondent à un apport de compost appliqué au sol de $0,10,20$ et 40 t.ha ${ }^{-1}$ respectivement.

Les incubations ont été réalisées à l'obscurité, à $28^{\circ} \mathrm{C}$ et à un taux d'humidité de $31 \%$, contrôlée et ajustée par pesée durant toute la période des incubations. Les mélanges compost + sol ont été homogénéisés et placés individuellement dans des bocaux en raison de 3 répétitions par dose. Un pilulier de $10 \mathrm{ml}$ d'eau a été mis à l'intérieur du bocal pour 
éviter le desséchement rapide des échantillons. Un pilulier contenant $10 \mathrm{ml}$ de $\mathrm{NaOH}(0,5 \mathrm{~N})$ a été aussi placé à l'intérieur du bocal pour piéger le $\mathrm{CO}_{2}$ produit par la respiration microbienne. Un bocal témoin a été inséré dans le dispositif pour mesurer le $\mathrm{CO}_{2}$ présent dans l'atmosphère. La différence de $\mathrm{CO}_{2}$ dégazé entre un bocal contenant les échantillons et le bocal témoin représente la quantité de $\mathrm{CO}_{2}$ issu de la respiration des microorganismes. Le dosage du $\mathrm{CO}_{2}$ a été fait par titrimétrie de la soude à $1,3,7,14,21$ et 28 jours après le début de l'incubation.

L'azote minéral a été dosé dans les échantillons à $0,1,3,7,14,21$ et 28 jours au cours de l'incubation décrite ci-dessus. Il a été extrait dans un mélange de $10 \mathrm{~g}$ d'échantillon frais de chaque bocal et $40 \mathrm{ml}$ de chlorure de potassium $\mathrm{KCl}(1 \mathrm{M})$. Après agitation pendant $1 \mathrm{~h}$ et centrifugation pendant $10 \mathrm{~min}$ à 4000 t/min, $3 \mathrm{ml} \mathrm{du}$ surnageant ont été prélevés pour doser l'azote minéral par colorimétrie en flux continu.

\section{Traitement statistique des données}

Les données recueillies ont été traitées à l'aide du logiciel XLSTAT version 5.0. Les tests de vérification de la normalité ont été effectués pour effectuer des tests statistiques paramétriques. Dans le cas où la normalité était respectée, les variantes étudiées étaient comparées par une ANOVA à un facteur fixe. Les comparaisons des moyennes ont été faites au seuil $\mathrm{p}=0,05$ à l'aide du test de Student Newman-Keul. Dans le cas où la normalité n'est pas respectée, le test non-paramétrique de Kruskal-Wallis était utilisé pour comparer les variantes. Pour comparer les variantes entre elles, le test de Dunn a été effectué sur les données.

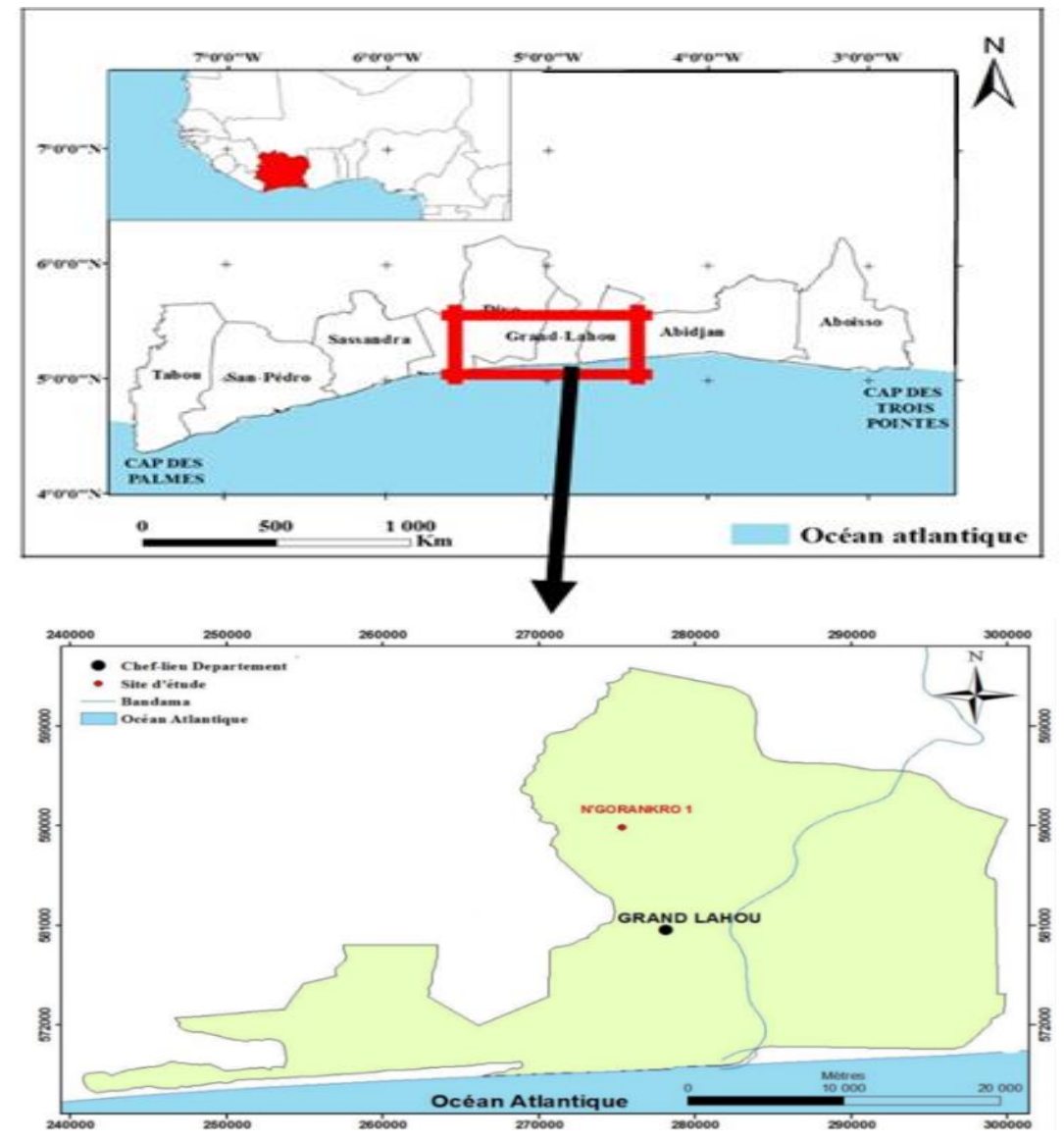

Figure 1 : Zone d'étude. 
Tableau 1 : Quantité de rafles et de fientes, rapport F/R par traitement.

\begin{tabular}{llll}
\hline Traitement & $\begin{array}{l}\text { Quantité de rafles } \\
\text { (kg de } \mathbf{M H )}\end{array}$ & $\begin{array}{l}\text { Quantité de fientes } \\
\text { (kg de } \mathbf{M H )}\end{array}$ & $\begin{array}{l}\text { Rapport fiente/rafles } \\
\text { (par traitement) }\end{array}$ \\
\hline T0 & 720 & 0 & 0 \\
T1 & 720 & 200 & 0,28 \\
T2 & 720 & 400 & 0,56 \\
T3 & 720 & 600 & 0,83 \\
\hline
\end{tabular}

MH : matière humide

Tableau 2 : Caractéristiques physico-chimiques du sol $(0-20 \mathrm{~cm})$.

\begin{tabular}{ll}
\hline Paramètres & Valeurs \\
\hline Argile (\%) & 9,5 \\
Limon (\%) & 13,3 \\
Sable (\%) & 77,15 \\
$\mathrm{pH}$ eau & 4,42 \\
$\mathrm{pH}$ & 3,83 \\
$\mathrm{C}\left(\mathrm{mg} \cdot \mathrm{kg}^{-1}\right)$ & 8030,25 \\
$\mathrm{~N}\left(\mathrm{mg} \cdot \mathrm{kg}^{-1}\right)$ & 588,59 \\
$\mathrm{P}\left(\mathrm{mg} \cdot \mathrm{kg}^{-1}\right)$ & 9,81 \\
$\mathrm{C} / \mathrm{N}$ & 13,67 \\
$\mathrm{MO}(\%)$ & 1,38 \\
$\mathrm{CEC}\left(\mathrm{cmol}^{(+)} \cdot \mathrm{kg}^{-1}\right)$ & 2,86 \\
$\mathrm{Ca}$ & 1,00 \\
$\mathrm{~K}^{+}\left(\mathrm{cmol}^{(+)} \cdot \mathrm{kg}^{-1}\right)$ & 0,1 \\
$\mathrm{Na}^{+}\left(\mathrm{cmol}^{(+)} \cdot \mathrm{kg}^{-1}\right)$ & 0,38 \\
\hline
\end{tabular}

\section{RESULTATS}

\section{Température}

L'évolution temporelle de la température a montré une tendance similaire quel que soit le traitement. Cependant, les températures les plus élevées ont été enregistrées avec $\mathrm{T} 3$, traitement ayant un grand rapport fiente/rafle (Figure 2). La tendance générale de l'évolution de la température présente trois phases. Lors de la première phase (jour 1 à 4), la température a augmenté de 27 à $35^{\circ} \mathrm{C}$ pour le $\mathrm{T} 0$ et de 30 à $60{ }^{\circ} \mathrm{C}$ pour les traitements $\mathrm{T} 1, \mathrm{~T} 2$ et $\mathrm{T} 3$. Pendant les retournements, une baisse de température a été observée. Elle a été caractérisée par les pics descendants. A partir de la deuxième semaine (seconde phase), les températures ont atteint les pics maximaux variables en fonction du rapport fiente/rafle dans le mélange initial. Ces pics maximaux étaient de $55{ }^{\circ} \mathrm{C}$ pour $\mathrm{T} 0$ (jour 13 ), $65{ }^{\circ} \mathrm{C}$ pour T1 (jour 19), $70{ }^{\circ} \mathrm{C}$ pour T2 (jour 37) et $73{ }^{\circ} \mathrm{C}$ pour T3 (jour 43 ). Après ces pics, il a été noté une baisse des températures au niveau des différents traitements pour se stabiliser à 29 ${ }^{\circ} \mathrm{C}$ pour $\mathrm{T} 0, \mathrm{~T} 1, \mathrm{~T} 2$ et $\mathrm{T} 3$ respectivement au $115,163,169$ et $181^{\mathrm{e}}$ jour. 
pH

Les valeurs instantanées de $\mathrm{pH}$ ont varié tout le long du processus de compostage pour se stabiliser à la maturité du compost avec un $\mathrm{pH}$ compris entre 7 et 8 . Le $\mathrm{pH}$ le plus élevé a été obtenu avec le traitement T3 et le plus faible avec le traitement T0. Cependant, les moyennes de $\mathrm{pH}$ des traitements T1, T2 et T3 sont statistiquement identiques à $\mathrm{p}<0,05$ et statistiquement différentes de celle du traitement $\mathrm{T} 0$ à $\mathrm{p}>0,05$, selon le test de Student Newman-Keuls (SNK). Les valeurs de $\mathrm{pH}$ mesurées en laboratoire pour ces quatre composts sont présentées à la Figure 3 et confirment celles obtenues pendant le processus. Quel que soit le traitement appliqué, les composts présentent un $\mathrm{pH}$ alcalin allant de 8,32 à 8,96. Le traitement $\mathrm{T} 2$ est perçu comme celui ayant le $\mathrm{pH}$ le plus basique $(\mathrm{pH}=8,96)$ et le traitement $\mathrm{T} 1$ celui qui a le $\mathrm{pH}$ le moins alcalin $(\mathrm{pH}=8,32)$. Les valeurs de $\mathrm{pH}$ des deux autres traitements sont comprises entre ces deux valeurs extrêmes. Il est à noter que durant le processus de compostage, les $\mathrm{pH}$ relevés ont varié entre 5 et 9,2.

\section{Humidité}

Contrairement à la température, l'humidité n'est pas corrélée au rapport fiente/rafle dans le tas initial. En effet, l'analyse des résultats de l'humidité massique mesurée en laboratoire a montré que les teneurs en eau varient de $58,2 \% \pm 2,03 \%$ dans le traitement $\mathrm{T} 1$ à $51,7 \% \pm 2,79 \%$ dans le traitement T2. Les traitements T0 $(57,23 \% \pm$ $1,52 \%)$ et $\mathrm{T} 3(55,36 \% \pm 0,34 \%)$ ont enregistré des teneurs moyennes d'humidité massique comprises entre ces deux valeurs (Figure 4). Ces résultats obtenus confirment ceux obtenus durant le processus de compostage avec une valeur moyenne de la teneur en eau de $56,4 \% \pm 2 \%$. La gamme de variation des teneurs en eau lors du compostage était de 40 à $60 \%$.

\section{Estimation des teneurs en nutriments dans les composts}

Les teneurs initiales en nutriments contenues dans les rafles et fientes brutes sont présentées dans le Tableau 3. Les résultats indiquent que ces matières sont riches en nutriments. Cependant, la fiente de volaille présente des teneurs plus élevées par rapport aux rafles de palmier $\left(\mathrm{N}: 28450 \mathrm{mg} \cdot \mathrm{kg}^{-1}, \mathrm{P}\right.$ : 17250 mg.kg-1, K: 43270 mg.kg-1 ${ }^{-1}$ Ca: $75405 \mathrm{mg} \cdot \mathrm{kg}^{-1}$ et $\left.\mathrm{Mg}: 14535 \mathrm{mg} \cdot \mathrm{kg}^{-1}\right)$. La comparaison des teneurs initiales avec les teneurs finales en nutriments après compostage est présentée dans le Tableau 4. Les résultats ont montré une baisse de la teneur des éléments $(\mathrm{N}, \mathrm{P}, \mathrm{Ca}, \mathrm{K}, \mathrm{Mg})$ lors du compostage. Cette perte est indiquée par une baisse de la teneur de ces nutriments dans les composts finaux. La perte en nutriment lors du compostage augmente quel que soit le nutriment du T0 au T3. La gamme de variation de la perte en nutriments varie de $4760 \mathrm{mg} \cdot \mathrm{kg}^{-1}$ à $14840 \mathrm{mg} \cdot \mathrm{kg}^{-1}$, de 2011,25 mg.kg ${ }^{-1}$ à 6327,5 mg.kg ${ }^{-1}$, de $9940 \mathrm{mg} \cdot \mathrm{kg}^{-1}$ à $19930 \mathrm{mg} \cdot \mathrm{kg}^{-1}$, de $3840 \mathrm{mg} \cdot \mathrm{kg}^{-1}$ à 14160 mg.kg ${ }^{-1}$ et de 1010 mg.kg-1 à 4140 mg.kg ${ }^{-1}$ respectivement pour le $\mathrm{N}, \mathrm{P}, \mathrm{K}$ Ca et $\mathrm{Mg}$. Dans les composts finaux, les teneurs les plus élevées ont été obtenues avec le compost T3 et les teneurs les plus faibles avec le compost $\mathrm{T} 0$. Les traitements $\mathrm{T} 1$ et $\mathrm{T} 2$ ont présentés des teneurs intermédiaires entre $\mathrm{T} 0$ et $\mathrm{T} 3$. Le rapport $\mathrm{C} / \mathrm{N}$ des rafles et de la fiente de volaille après dosage du carbone est respectivement de 65,55 et 13,65. La comparaison des rapports $\mathrm{C} / \mathrm{N}$ initiaux et des rapports $\mathrm{C} / \mathrm{N}$ finaux dans les différents traitements montre une diminution de ce rapport quel que soit le traitement avec un rapport $\mathrm{C} / \mathrm{N}$ plus élevé avec $\mathrm{T} 0(15,12)$ et plus faible avec T2 $(14,07)$.

\section{Estimation des teneurs en éléments traces métalliques (ETM) dans les composts}

Les teneurs initiales en chrome $(\mathrm{Cr})$, zinc $(\mathrm{Zn})$ et plomb $(\mathrm{Pb})$ contenues dans les rafles et fientes brutes sont présentées dans le Tableau 5. Les résultats indiquent que les matières brutes de départ étaient chargées en Cr (450 mg. $\mathrm{kg}^{-1}$ pour les rafles et $309 \mathrm{mg} \cdot \mathrm{kg}^{-1}$ pour la fiente de volaille) et faiblement chargées en $\mathrm{Pb}$ et $\mathrm{Zn}\left(\mathrm{Pb}: 15 \mathrm{mg} \cdot \mathrm{kg}^{-1}, \mathrm{Zn}: 33\right.$ $\mathrm{mg} \cdot \mathrm{kg}^{-1}$ pour les rafles et $\mathrm{Pb}: 85,5 \mathrm{mg} \cdot \mathrm{kg}^{-1}$ pour la fiente). Cependant, la fiente de volaille présente des teneurs très élevées en Zn par rapport aux rafles de palmier (656 mg. $\left.\mathrm{kg}^{-1}\right)$. La comparaison des teneurs initiales avec les teneurs finales montre une baisse de 
la teneur des éléments lors du compostage excepté le $\mathrm{Pb}$ et le $\mathrm{Zn}$ où on n'observe pas de variation avec T0 (Figure 5). Cette baisse augmente avec la quantité de fiente apportée dans les différents traitements quel que soit l'élément, du T0 à T3. Le pourcentage de perte était de 88,55 à 95,39\%, de 73,31 à $83 \%$ et de 81,48 à $87,22 \%$ respectivement pour le $\mathrm{Cr}, \mathrm{Pb}$ et $\mathrm{Zn}$. Malgré ces variations, les teneurs obtenues en fin de compostage sont faibles comparées aux normes françaises établies.

\section{Minéralisation de carbone}

La Figure 6 montre l'évolution du flux et la quantité cumulée de $\mathrm{C}-\mathrm{CO}_{2}$ émis par les différents mélanges compost + sol pour une période de 28 jours. Les évolutions du flux et de la quantité de $\mathrm{C}-\mathrm{CO}_{2}$ émis au cours de la période d'incubation présentent des allures similaires quelle que soit la dose de compost apportée. Les mélanges compost + sol ont induit des augmentations de flux et de quantités cumulées de $\mathrm{C}-\mathrm{CO}_{2}$ comparativement au témoin. Les quantités de carbone des différentes doses exprimées en $\mathrm{mg} \mathrm{C} / \mathrm{kg}$ de sol sec se présentent selon l'ordre décroissant suivant : D3 > D2 > D1 > D0. Pour les différentes doses, les flux de $\mathrm{C}-\mathrm{CO}_{2}$ présentent un pic à 1 jour d'incubation puis décroissent au cours du temps (Figure 6A). Cette diminution de la quantité de $\mathrm{C}-\mathrm{CO}_{2}$ tend vers une stabilisation de la production de $\mathrm{C}$ $\mathrm{CO}_{2}$. A la fin de l'incubation, la production de carbone par les sols contenant les apports de compost commence à se rapprocher de celle du témoin. D'après le test Student NewmannKeuls, il n'y a pas de différence significative entre les quantités de $\mathrm{C}-\mathrm{CO}_{2}$ obtenues avec les doses D0, D1et D2. Par contre, les quantités obtenues avec la dose D3 sont significativement différentes des autres. La Figure 6B représente la production cumulée de $\mathrm{C}-\mathrm{CO}_{2}$ de toutes les doses tout au long de la période d'incubation. L'analyse des graphes traduisant les flux de minéralisation et les quantités cumulées de $\mathrm{C}-\mathrm{CO}_{2}$ émises révèle deux phases de minéralisation. Une phase de minéralisation rapide caractérisée par une forte pente observée pendant les premiers jours et une phase de minéralisation lente caractérisée par un adoucissement de façon à ce que la courbe tende vers un palier à la fin de l'expérience. Ceci confirme le ralentissement de la vitesse de la minéralisation du carbone observée par la diminution de la production journalière de $\mathrm{C}$ $\mathrm{CO}_{2}$. Cependant, la quantité cumulée de $\mathrm{CO}_{2}$ libéré dans les sols avec ajout de compost est supérieure à celle du témoin $\mathrm{D} 0$ passant de $316,05 \mathrm{mg}$ C-CO $\mathrm{kg}^{-1}$ sol (témoin D0) à

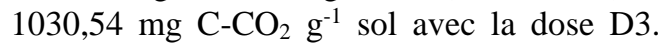
Les doses $\mathrm{D} 1$ et $\mathrm{D} 2$ étant intermédiaires à ces valeurs extrêmes. Après 28 jours d'incubation, $3,93 \%, 5,69 \%, 6,20 \%$ et $7,79 \%$ du carbone organique total sont minéralisé respectivement avec les doses D0, D1, D2 et D3 (Figure 7). D'après les tests statistiques, il n'y a pas de différence significative entre les quantités de C- $\mathrm{CO}_{2}$ obtenues avec les doses D0, D1et D2. Par contre, les quantités obtenues avec la dose D3 sont significativement différentes des autres.

\section{Minéralisation de l'azote}

L'évolution de l'azote minéral dans les différents mélanges au cours de la période d'incubation est présentée à la Figure 8. Les résultats révèlent que la quantité d'azote minéral varie selon la dose de compost apporté au sol avec D3>D2>D1>D0. Des variations de la quantité d'azote minéral sont observées dans les mélanges. Les quantités d'N minéral avec la dose D1 ont diminué dans la période du $1^{\mathrm{er}}$ au $3^{\mathrm{e}}$ jour d'incubation et par la suite elles ont augmenté. De même, les quantités d'N minéral avec les doses D2 et D3 ont diminué dans la période du $7^{\mathrm{e}}$ au $14^{\mathrm{e}}$ jour d'incubation. Cependant, avec la dose D0, la quantité d'azote minéral a augmenté au cours au temps.

Après 28 jours d'incubation, la quantité d'azote minéral obtenue en fonction des doses est de 79,6 mg.kg-1 avec D0 ; $122,78 \mathrm{mg} \cdot \mathrm{kg}^{-1}$ avec D1 ; 185,78 mg.kg ${ }^{-1}$ avec D2 et $301,46 \mathrm{mg} \cdot \mathrm{kg}^{-1}$ avec D3. D'après les tests statistiques, il n'y a pas de différence significative entre les quantités d'N minéral obtenues avec les doses D0, D1et D2. Par contre, les quantités obtenues avec la dose D3 sont significativement différentes des autres. 


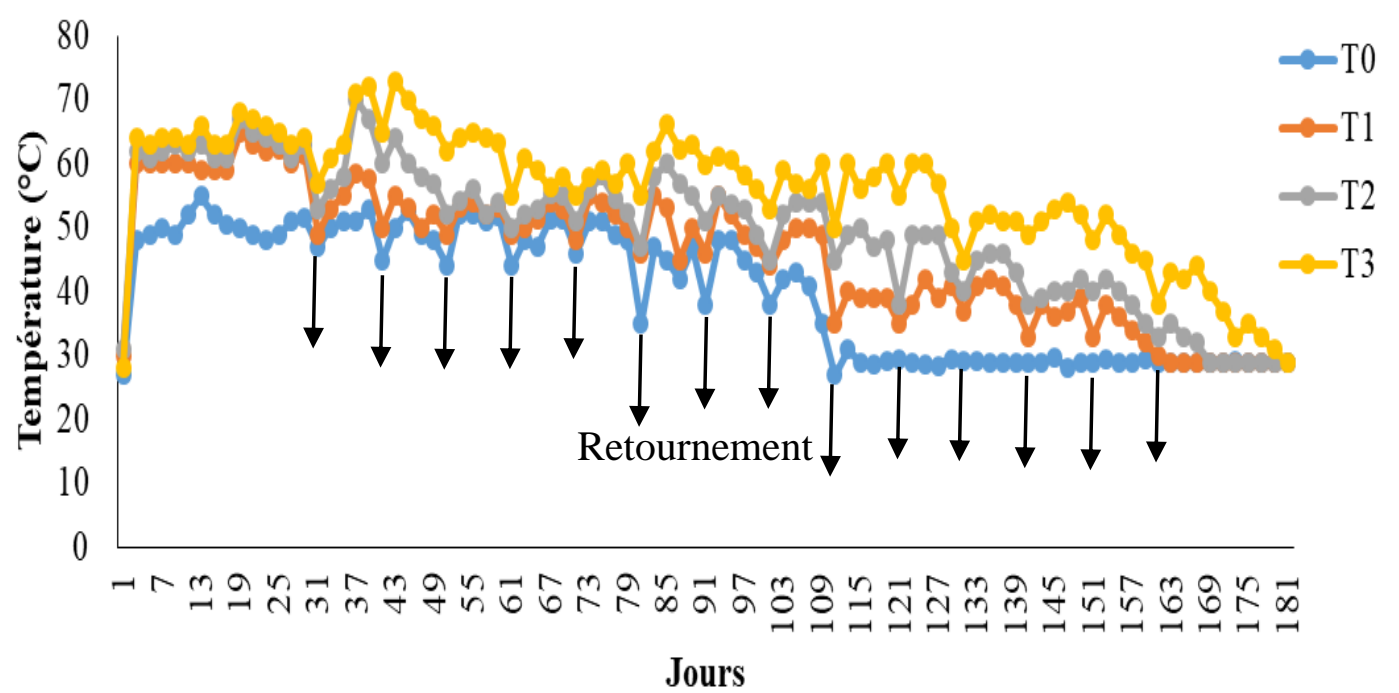

Figure 2: Courbe évolutive de la température durant la période de compostage (T0 $=720 \mathrm{~kg}$ de rafles $+0 \mathrm{~kg}$ de fiente de volaille; $\mathrm{T} 1=720 \mathrm{~kg}$ de rafles $+200 \mathrm{~kg}$ de fiente de volaille; $720 \mathrm{~kg}$ de rafles $+400 \mathrm{~kg}$ de fiente de volaille; $720 \mathrm{~kg}$ de rafles $+600 \mathrm{~kg}$ de fiente de volaille).

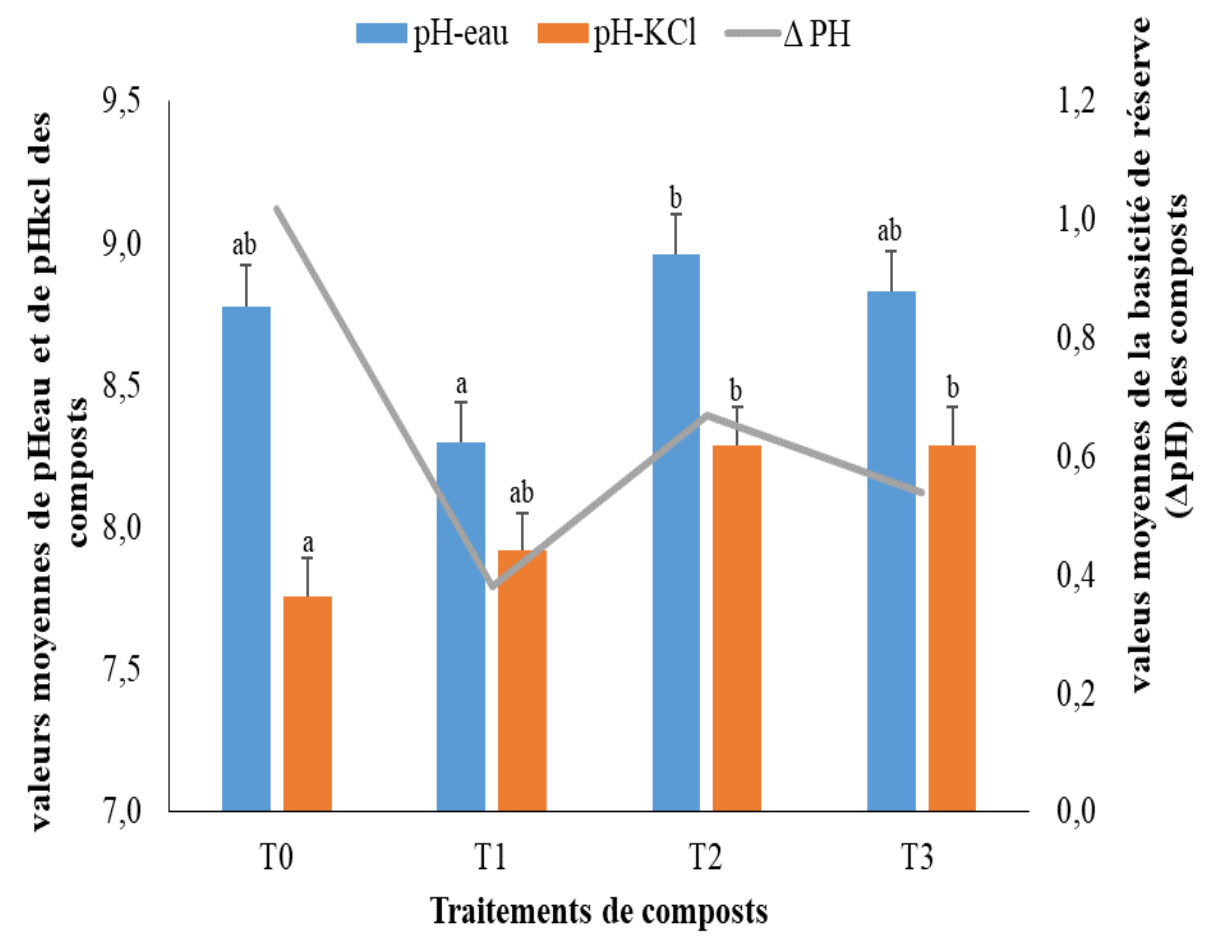

Figure $3: \mathrm{pH}$ des composts en fonction des traitements $(\mathrm{T} 0=720 \mathrm{~kg}$ de rafles $+0 \mathrm{~kg}$ de fiente de volaille $; \mathrm{T} 1=720 \mathrm{~kg}$ de rafles $+200 \mathrm{~kg}$ de fiente de volaille $; 720 \mathrm{~kg}$ de rafles $+400 \mathrm{~kg}$ de fiente de volaille $; 720 \mathrm{~kg}$ de rafles $+600 \mathrm{~kg}$ de fiente de volaille). 


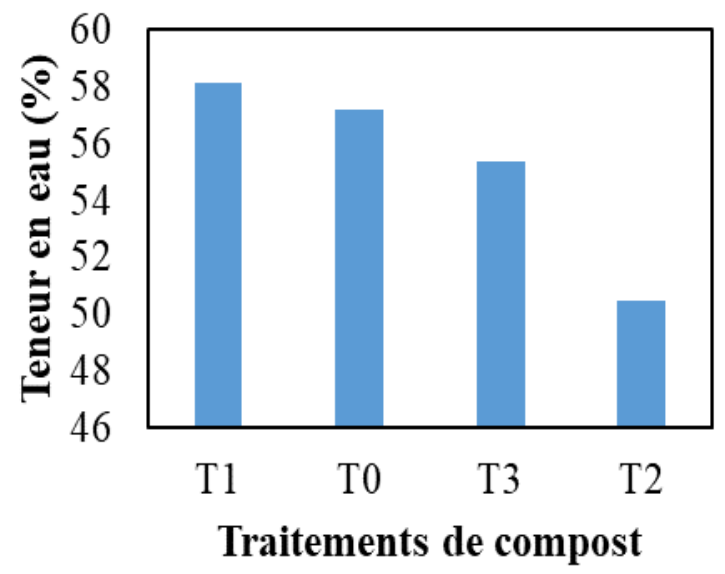

Figure 4 : Teneurs moyennes en eau selon les traitements. (T0 $=720 \mathrm{~kg}$ de rafles $+0 \mathrm{~kg}$ de fiente de volaille ; $\mathrm{T} 1=720 \mathrm{~kg}$ de rafles $+200 \mathrm{~kg}$ de fiente de volaille $; 720 \mathrm{~kg}$ de rafles $+400 \mathrm{~kg}$ de fiente de volaille $; 720 \mathrm{~kg}$ de rafles +600 $\mathrm{kg}$ de fiente de volaille).

Tableau 3 : Teneurs $\left(\mathrm{mg} \mathrm{kg}^{-1}\right)$ en éléments nutritifs des matières brutes.

\begin{tabular}{llllllll}
\hline & $\mathbf{C}$ & $\mathbf{N}$ & $\mathbf{P}$ & $\mathbf{K}$ & $\mathbf{C a}$ & $\mathbf{M g}$ & $\mathbf{C} / \mathbf{N}$ \\
\hline Rafle & 442500 & 6750 & 2450 & 26900 & 8495 & 4380 & 65,55 \\
Fiente & 387700 & 28450 & 17250 & 43270 & 75405 & 14535 & 13,65 \\
\hline
\end{tabular}

Tableau 4 : Teneurs initiales et finales en nutriments dans les composts en fonction des traitements.

\begin{tabular}{llllll}
\hline & & T0 & T1 & T2 & T3 \\
\hline & $\mathrm{C}$ & $442500 \mathrm{~d}$ & $617633,47 \mathrm{c}$ & $792766,94 \mathrm{~b}$ & $967900,41 \mathrm{a}$ \\
Teneurs initiales & $\mathrm{N}$ & $6750 \mathrm{~d}$ & $19722,01 \mathrm{c}$ & $32694,02 \mathrm{~b}$ & $45666,04 \mathrm{a}$ \\
$\left(\right.$ mg.kg $\left.^{-1}\right)$ & $\mathrm{P}$ & $2450 \mathrm{~d}$ & $10366,45 \mathrm{c}$ & $18282,91 \mathrm{~b}$ & $26199,37 \mathrm{a}$ \\
& $\mathrm{K}$ & $26900 \mathrm{~d}$ & $46561,59 \mathrm{c}$ & $66223,19 \mathrm{~b}$ & $85884,79 \mathrm{a}$ \\
& $\mathrm{Ca}$ & $8495 \mathrm{~d}$ & $42661,97 \mathrm{c}$ & $76828,95 \mathrm{~b}$ & $110995,93 \mathrm{a}$ \\
& $\mathrm{Mg}$ & $4380 \mathrm{~d}$ & $11070,93 \mathrm{c}$ & $17761,87 \mathrm{~b}$ & $24452,81 \mathrm{a}$ \\
& $\mathrm{C} / \mathrm{N}$ & $65,55 \mathrm{a}$ & $31,35 \mathrm{~b}$ & $24,28 \mathrm{c}$ & $21,22 \mathrm{~d}$ \\
\cline { 2 - 6 } & $\mathrm{C}$ & $72000 \mathrm{~d}$ & $146900 \mathrm{c}$ & $197000 \mathrm{~b}$ & $213300 \mathrm{a}$ \\
Teneurs finales & $\mathrm{N}$ & $4760 \mathrm{c}$ & $9800 \mathrm{~b}$ & $14000 \mathrm{a}$ & $14840 \mathrm{a}$ \\
$\left(\mathbf{m g . k g}{ }^{-1}\right)$ & $\mathrm{P}$ & $2011,25 \mathrm{~d}$ & $3725 \mathrm{c}$ & $5871,25 \mathrm{~b}$ & $6327,5 \mathrm{a}$ \\
& $\mathrm{K}$ & $9940 \mathrm{~d}$ & $13190 \mathrm{c}$ & $18700 \mathrm{~b}$ & $19930 \mathrm{a}$ \\
& $\mathrm{Ca}$ & $3840 \mathrm{~d}$ & $8540 \mathrm{c}$ & $12380 \mathrm{~b}$ & $14160 \mathrm{a}$ \\
& $\mathrm{Mg}$ & $1010 \mathrm{~d}$ & $2390 \mathrm{c}$ & $3500 \mathrm{~b}$ & $4140 \mathrm{a}$ \\
& $\mathrm{C} / \mathrm{N}$ & $15,12 \mathrm{a}$ & $14,98 \mathrm{a}$ & $14,07 \mathrm{~b}$ & $14,37 \mathrm{ab}$ \\
\hline
\end{tabular}

(Les données sur la même ligne suivies de la même lettre ne sont pas significativement différentes selon le test de Newmankeuls $\mathrm{p}<0,05)$.

Tableau 5 : Teneurs $\left(\mathrm{mg} \cdot \mathrm{kg}^{-1}\right)$ en ETM dans les substrats bruts.

\begin{tabular}{|c|c|c|c|}
\hline & $\mathrm{Cr}$ & $\mathbf{P b}$ & Zn \\
\hline Rafle brute & 450 & 15 & 33 \\
\hline Fiente brute & 309 & 85,5 & 656 \\
\hline $\begin{array}{lrr}\text { Valeurs } & \text { seuils } & (\mathrm{ppm}) \\
\text { AFNOR, }(2005) & \\
\end{array}$ & 200 & 180 & 300 \\
\hline
\end{tabular}




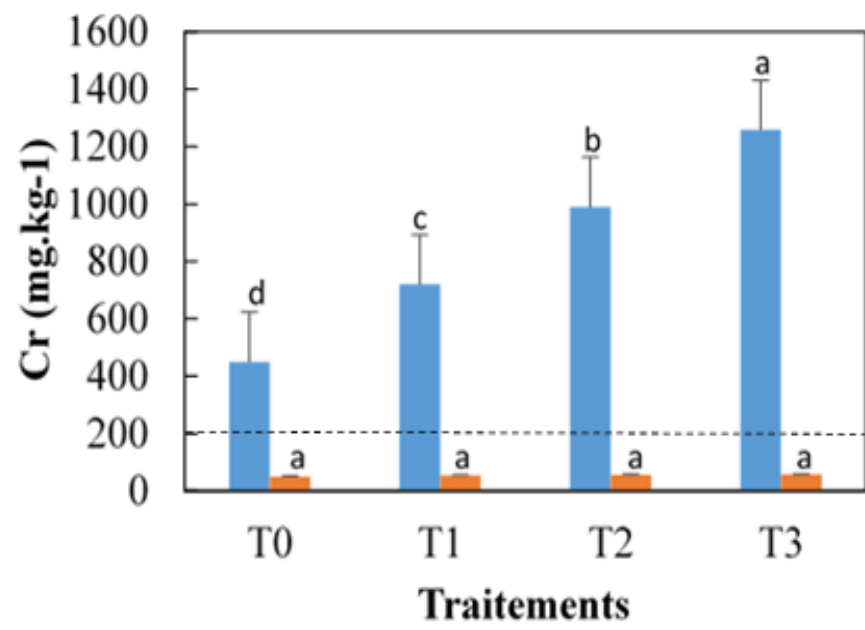

- Teneur initiale

- Teneur finale

-.-.-Valeurs seuils

(AFNOR, 2005)

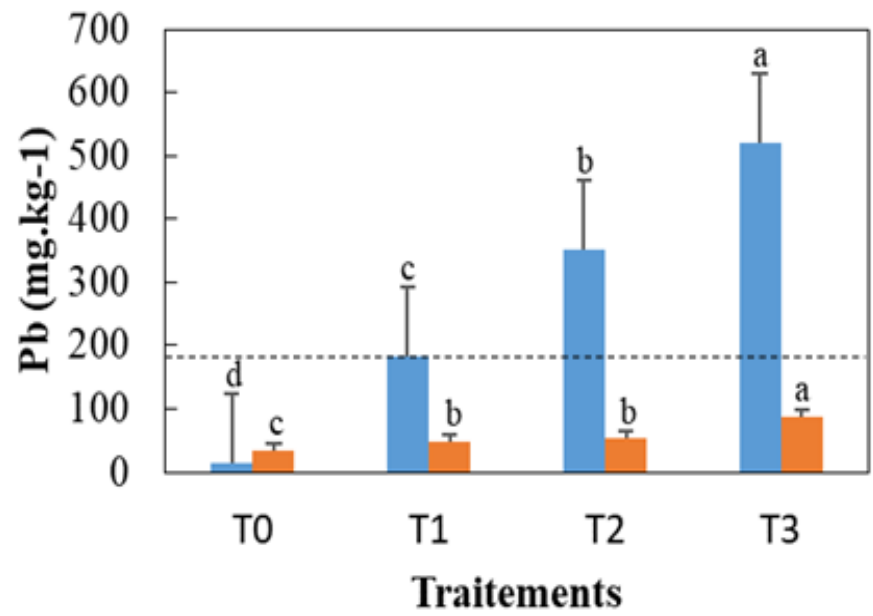

- Teneur initiale

- Teneur finale

-...-Valeurs seuils

(AFNOR, 2005)

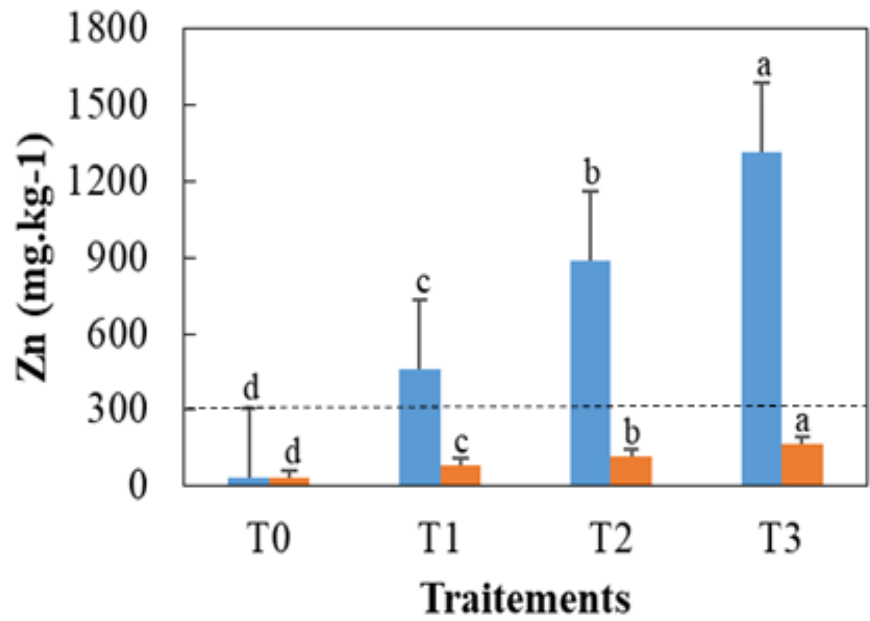

a Teneur initiale

- Teneur finale

-.--Valeurs seuils

(AFNOR, 2005)

Figure 5 : Teneurs initiales et finales en $\mathrm{Cr}, \mathrm{Pb}$ et $\mathrm{Zn}$ dans les composts en fonction des traitements. ( $\mathrm{T} 0=720 \mathrm{~kg}$ de rafles $+0 \mathrm{~kg}$ de fiente de volaille $; \mathrm{T} 1=720 \mathrm{~kg}$ de rafles $+200 \mathrm{~kg}$ de fiente de volaille $; 720 \mathrm{~kg}$ de rafles + $400 \mathrm{~kg}$ de fiente de volaille; $720 \mathrm{~kg}$ de rafles $+600 \mathrm{~kg}$ de fiente de volaille). 

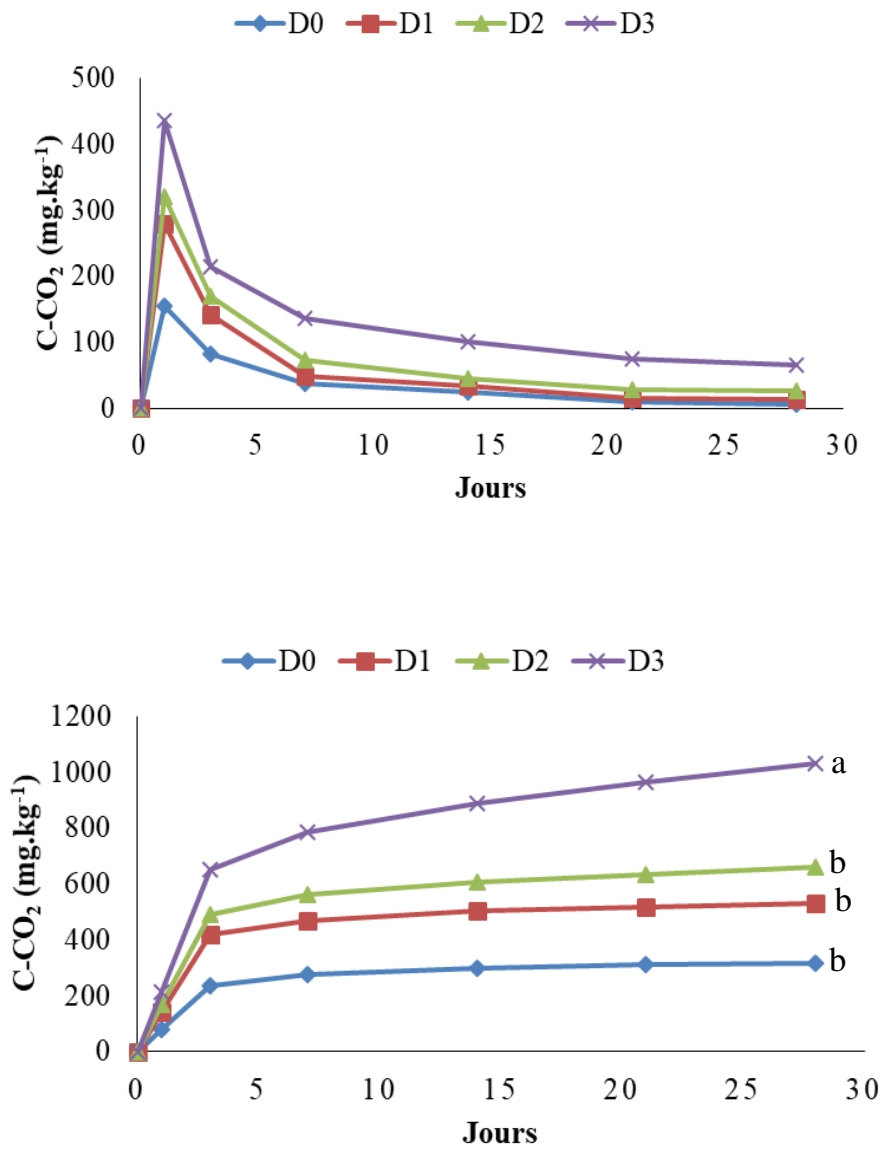

Figure 6: Cinétique. de la minéralisation du carbone au cours du temps, (A) journalière ; (B) cumulée $\left(\mathrm{D} 0=0\right.$ t.ha ${ }^{-1} ; \mathrm{D} 1=10$ t.ha $^{-1} ; \mathrm{D} 2=20$ t.ha $^{-1} ; \mathrm{D} 3=40$ t.ha $\left.{ }^{-1}\right)$.

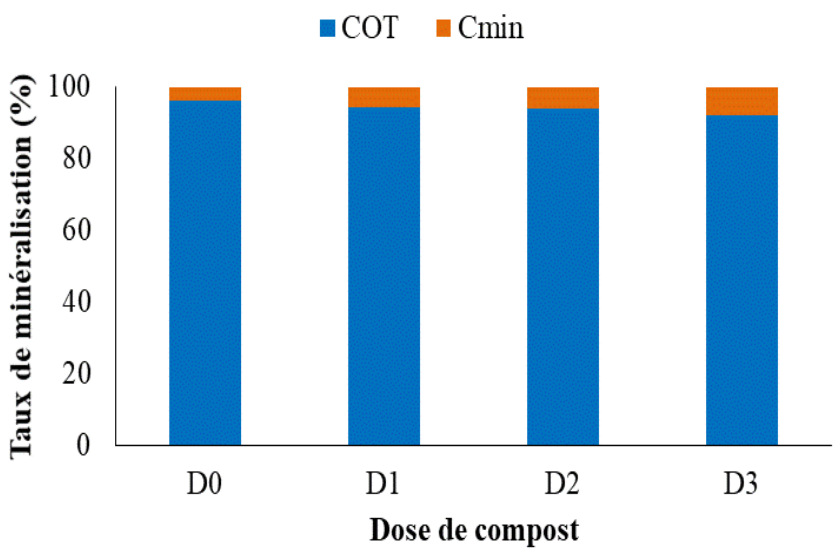

Figure 7 : Quantité de carbone minéralisé après 28 jours d'incubation $\left(\mathrm{D} 0=0\right.$ t.ha ${ }^{-1} ; \mathrm{D} 1=10$ t.ha $^{-1}$; D2 $=20$ t.ha $^{-1} ;$ D3= 40 t.ha $^{-1}$; COT : carbone organique total ; Cmin : carbone minéralisé). 


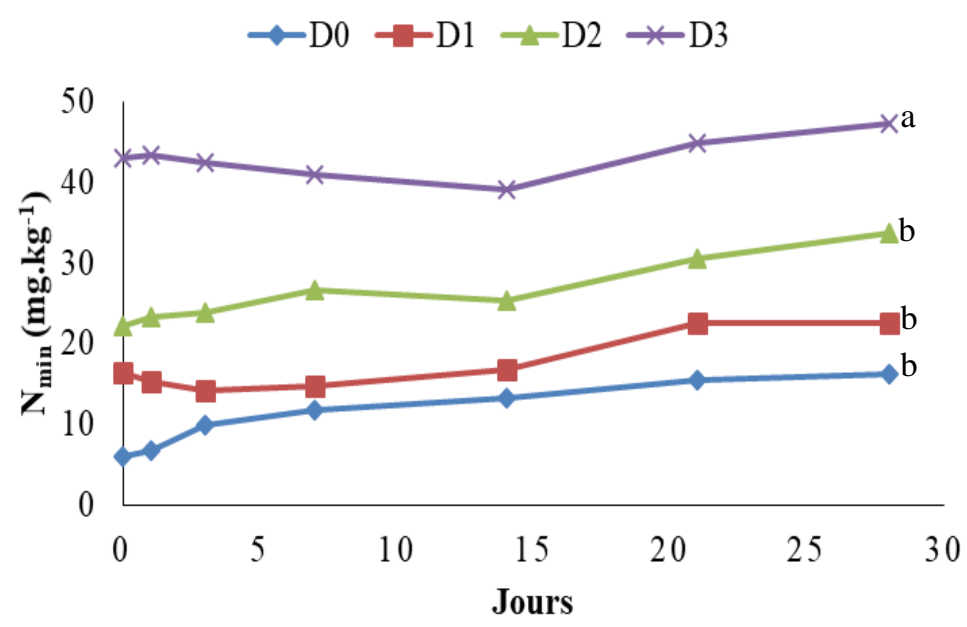

Figure 8 : Evolution de l'azote minéral (Nmin) après 28 jours d'incubation.

\section{DISCUSSION Température}

Le compostage de la plupart des substrats est caractérisé par une période initiale de dégradation rapide suivie d'une plus longue période de dégradation lente (Diaz et al., 2002). Quatre jours après la mise en tas des composts, une augmentation de la température a été observée. Cette augmentation atteignait des valeurs de $35{ }^{\circ} \mathrm{C}$ pour $\mathrm{T} 0$ et $60{ }^{\circ} \mathrm{C}$ pour les traitements $\mathrm{T} 1, \mathrm{~T} 2$ et T3. Deux (2) autres pics de température avaient été atteints; il s'agit du pic de $55{ }^{\circ} \mathrm{C}$ pour le plus faible et du pic de $73{ }^{\circ} \mathrm{C}$ pour le plus élevé. Ces résultats corroborent ceux obtenus par Misra et al. (2005) et Baharuddin et al. (2009) qui au cours de leurs travaux ont observé que quatre jours après la mise en place du compostage, on observe une hausse de la température qui correspond à la phase thermophile. En effet, selon ces auteurs, les conditions (humidité, taux d'oxygène lacunaire, température...) étaient favorables pour la dégradation de la matière et, la hausse de la température traduit la décomposition de la matière organique fraîche sous l'action des bactéries et des champignons. Cependant, la montée de la température était moins importante dans le compost $\mathrm{T} 0$ contrairement à celle observée dans les composts $\mathrm{T} 1, \mathrm{~T} 2$ et
T3. Le compost T0 est exclusivement composé de rafles de palmier alors que les composts $\mathrm{T} 1, \mathrm{~T} 2$ et $\mathrm{T} 3$ contiennent un ajout de fiente comme activateur. Cet activateur serait à l'origine des intenses activités microbiennes qui ont engendré des augmentations importantes des températures dans les traitements. Après cette phase de réchauffement, les températures ont diminué régulièrement jusqu'à $29^{\circ} \mathrm{C}$. L'abaissement de la température subséquent s'expliquerait par une diminution de la matière organique biodégradable et la mort de micro-organismes thermophiles (Hassen et al., 2001). Aussi, au cours de cette phase les micro-organismes mésophiles colonisent à nouveau le compost.

\section{Rôle du pH dans le processus du compostage}

Les $\mathrm{pH}$ relevés durant le processus de compostage ont varié entre 5 et 9,2. Des résultats similaires ont été obtenus par Ben Ayed et al. (2005) et Koledzi (2011) Pour ces auteurs, le $\mathrm{pH}$ des suspensions solides (déchets en phase de dégradation aérobie, compost mûr) varie entre 5 et 8 et peut atteindre 9. En effet, une phase acidogène se produit au début du processus de dégradation par les bactéries acidogènes, décomposeurs du carbone complexe, provoquant ainsi une 
diminution du $\mathrm{pH}$ initial. La seconde phase correspond à une alcalinisation qui est une hydrolyse bactérienne de l'azote avec production d'ammoniac $\left(\mathrm{NH}_{3}\right)$ associée à la dégradation de protéines et à la décomposition d'acides organiques (Record, 2002 ; Mérigout, 2006).

\section{Humidité dans le processus de compostage}

Ce paramètre a évolué autour de l'optimum qui est de 40 à $60 \%$ tel que décrit par Misra et al. (2005). Les teneurs en eau correspondent donc à celles recommandées pour un processus normal de compostage pour un produit de meilleure qualité (Zorpas et al., 2003). En effet, il est indiqué qu'une bonne humidité favorise la mobilité et la croissance des micro-organismes décomposeurs (Francou, 2003), ce qui pourrait être le cas observé dans le processus de compostage des rafles de palmier. De plus, les phases de retournement opérées ont permis de maintenir une aération suffisante à la décomposition aérobie pour les tas des composts.

\section{Teneur en éléments majeurs $(\mathrm{N}, \mathrm{P}, \mathrm{K}, \mathrm{Mg}$ et $\mathrm{Ca}$ ) des composts et rapport $\mathrm{C} / \mathrm{N}$}

L'incorporation d'une quantité importante croissante de fiente de volaille dans les rafles avait pour but d'enrichir suffisamment le produit en fin de compostage. Le rapport $\mathrm{C} / \mathrm{N}$ des combinaisons initiales aurait permis d'atteindre un rapport $\mathrm{C} / \mathrm{N}$ favorable au processus de compostage (Misra et al., 2005). Selon ces auteurs, le rapport optimal $\mathrm{C} / \mathrm{N}$ des composts initiaux devrait situer entre 25 et 30 bien que des rapports situés entre 20 et 40 soient aussi acceptables. Lorsque le $\mathrm{C} / \mathrm{N}$ est supérieur à 40, la croissance des micro-organismes est limitée et implique une durée de compostage plus longue. Un rapport $\mathrm{C} / \mathrm{N}$ inférieur à 20 entraine une sous-utilisation de l'azote et le surplus d'azote peut être alors perdu dans l'atmosphère sous forme d'ammoniac ou d'oxyde nitreux. Dans les composts réalisés, le rapport $\mathrm{C} / \mathrm{N}$ a varié de 14,07 à 15,12 . Cette faible valeur traduirait la relative rapide minéralisation de l'azote et par conséquent sa disponibilité dans les composts (Biékré et al.,
2018). Plusieurs auteurs (Charnay, 2005 ; Misra et al., 2005 ; Huang et al., 2006) ont montré dans leurs travaux que le rapport $\mathrm{C} / \mathrm{N}$ est un indicateur de la maturité et de la qualité du compost. Pour ces auteurs, le rapport final $\mathrm{C} / \mathrm{N}$ en fin de compostage devrait se situer entre 10 et 15 . Ce qui est en adéquation avec les résultats obtenus dans notre étude. Par ce processus, des résultats similaires ont été obtenus par Debril et al. (2005) sur la gestion des déchets de Jussie par compostage. Ils ont montré une variation des teneurs en $\mathrm{N}, \mathrm{P}$ et $\mathrm{K}$ dans les composts. D’un point de vue général, les composts élaborés se trouvent être riche en nutriment. De ces résultats on peut déduire que les rafles de palmier sont très riches en éléments nutritifs, ce qui pourrait être bénéfique pour les sols et les plantes. Ces résultats corroborent les travaux de Singh et al. (2010) et Jacquemard (2012) qui ont montré que les rafles de palmier étaient riches en nutriments. Aussi, la quantité d'éléments nutritifs contenue dans les fientes aurait permis d'augmenter les teneurs en $\mathrm{N}, \mathrm{P}, \mathrm{K}$, $\mathrm{Mg}$ et $\mathrm{Ca}$ contenues dans les rafles. Cette richesse en éléments nutritifs des rafles brutes pourrait aussi être due à la fertilisation apportée dans les plantations de palmier à huile. En fonction du mélange des matériaux à composter, la quantité de chaque matériau pourrait avoir un effet significatif sur les teneurs en éléments nutritifs. Ainsi, dans notre cas le compost T3 comportant la quantité la plus élevée de fiente de volaille s'avère être le plus riche en nutriments par rapport aux trois autres composts (T0, T1 et T2). Ces résultats corroborent les travaux de Toundou (2016) et Biaou et al. (2017). Toutefois, des pertes en nutriments ont été observées à la fin du compostage ce qui pourrait être lié à la volatilisation de l'ammoniac concernant l'azote et à un lessivage concernant les autres éléments. Ces processus pourraient être causés par le retournement des tas ou lors des arrosages. Selon Weill et Duval (2009) et GAB 44 (2015) les pertes en azote augmentent avec la durée du compostage et le nombre de retournements. L'augmentation du $\mathrm{pH}$ pourrait entrainer une perte de l'azote durant la phase de compostage en particulier 
pour les fumiers de volaille (Weill et Duval, 2009).

\section{Teneur en éléments traces métalliques $(\mathrm{Pb}$, $\mathrm{Zn}$ et $\mathrm{Cr}$ ) des composts}

Les teneurs en $\mathrm{Pb}, \mathrm{Zn}$ et $\mathrm{Cr}$ dans les composts élaborés sont inférieures aux normes françaises. Par contre, des teneurs élevées ont été observées en $\mathrm{Cr}$ et en $\mathrm{Zn}$ dans les matériaux de départ. Ce qui suppose que le compostage aurait réduit les teneurs des métaux contenus dans les différents composts. En effet, selon Pakou et al. (2009), le compostage réduit la disponibilité de métaux présents dans les déchets organiques. Selon Amir et al. (2005), au cours du compostage, le taux des ETM tend à diminuer par lessivage suite à leur libération par décomposition des structures organiques. Ainsi, l'amendement des sols avec du compost peut être bénéfique pour les cultures alimentaires. Néanmoins, il devrait être contrôlé dans la mesure où les applications répétées des composts pourraient avoir par accumulation de polluants, un effet perturbateur sur le fonctionnement biologique des sols (Houot et al., 2009).

\section{Cinétiques de la minéralisation du carbone et de l'azote}

Les résultats de cette étude montrent deux phases d'émission de $\mathrm{CO}_{2}$ suggérant des cinétiques variables de minéralisation de la matière organique au cours de la période d'incubation. Des tendances similaires ont été observées dans la littérature par Khalil et al. (2005). Ces études soulignent la forte intensité de minéralisation de la matière organique durant les premiers jours d'incubation, indiquée par les pics de flux de $\mathrm{C}-\mathrm{CO}_{2}$ qui résulteraient de la décomposition des composés facilement dégradables contenus dans les apports organiques (Lim et Zaharah, 2002 ; Saletes et al., 2004). Selon Francou (2003), les cinétiques de minéralisation du carbone sont fonction du degré de maturité des matières organiques. L'auteur définit une gamme de stabilité de la matière organique. Il distingue les matières fortement stables ( 0 $10 \%)$, les matières stables $(10-15 \%)$, les matières moyennement stables (15-20\%), les matières instables (20-30\%) et les matières très instables $(>30 \%)$. Ainsi, dans notre étude, le compost obtenu qui a eu une cinétique de minéralisation très faible (D0 :3,93\%; D1 : $5,69 \%$, D2: $6,20 \%$ et D3: 7,79\%) est hautement stable. En plus, Elherradi et al. (2003) ont montré que les activités des microorganismes ont un effet «flush» de minéralisation entre 0 et 14 jours après leur incubation. En effet, sous l'effet d'une réhumectation des sols après séchage, il se produit un «flush » de minéralisation suivi de la diminution de la vitesse de minéralisation. Selon ces auteurs, ce flush correspond essentiellement à la biomasse microbienne détruite en partie par séchage ou à une matière organique très labile susceptible d'être minéralisée rapidement. En effet, avant d'être incubés, les échantillons de sol et de compost ont subi un desséchement puisqu'ils ont subi différents traitements (transport, tamisage, stockage). Cette dessication aurait pour conséquence une forte mortalité microbienne donnant lieu à une augmentation de la quantité de molécules organiques. Après l'humectation des échantillons, il y a donc une reprise de l'activité microbienne avec comme corollaire une production de $\mathrm{CO}_{2}$ issue de la minéralisation rapide des molécules organiques. Après avoir atteint une valeur maximale au cours des premiers jours, le $\mathrm{C}$ $\mathrm{CO}_{2}$ diminue progressivement avec le temps traduisant une diminution de l'activité microbienne due à l'épuisement du stock de molécules organiques suite à leur consommation pour leur métabolisme.

L'allure plate des courbes temporelles montre une faible dynamique de l'azote minéral dans les différentes doses. Ceci pourrait s'expliquer par une faible activité microbienne donc une bonne maturité des composts. Le taux de minéralisation de l'azote montre une stabilité temporelle malgré quelques légères variations. Des études d'incubation montrent que l'azote minéralisé peut être piégé par les constituants du sol ou temporairement immobilisé par les microorganismes car l'apport de matière organique au sol stimule la croissance et la reproduction microbiennes et, par conséquent, 
une concurrence accrue pour les nutriments disponibles dont l'azote (Azeez et Van Averbeke, 2010). De plus, selon Pansu et al. (2003), la transformation de l'azote organique incorporé au sol dépend non seulement de la biomasse microbienne mais aussi des caractéristiques biochimiques de la matière organique exogène. La cinétique de l'azote minéral est corrélée à l'apport des différentes doses de compost au sol (Elherradi et al., 2003). En effet le taux d'azote minéral le plus élevé a été obtenu avec D3 et le plus faible avec D0. Ce qui est en accord avec certains travaux qui ont montré que l'effet du compost sur la minéralisation de l'azote varie selon la quantité de compost appliquée (Masunga et al., 2016).

\section{Conclusion}

Cette étude a été initiée dans le but d'évaluer l'impact des apports croissants de fientes de volaille sur la valeur agronomique d'un compost de rafles de palmier à huile. Les résultats obtenus ont montré que les 4 types de composts élaborés présentent des teneurs élevées en nutriments et de faibles teneurs en ETM. Dans nos conditions d'expérimentation, l'apport croissant de fientes de volaille a amélioré de façon croissante la qualité des composts par une augmentation des teneurs en nutriments. Ainsi, le compost T3 composé de $720 \mathrm{~kg}$ de rafles et $600 \mathrm{~kg}$ de fientes de volaille semble être le plus efficient pour un amendement des sols pauvres en matières organiques.

\section{CONFLIT D'INTERETS}

Il n'y a pas de conflit d'intérêt entre les auteurs de cet article.

\section{CONTRIBUTIONS DES AUTEURS}

Tous les auteurs ont participé à la conception de l'étude, à l'expérimental et à la rédaction scientifique de l'article.

\section{REMERCIEMENTS}

Ce projet a été réalisé avec le soutien du projet PARFAO initié par l'AUF en collaboration avec l'IRD, de l'Agence Nationale d'Appui au Développement Rural
(ANADER) de Grand-Lahou dans le cadre de la mise en œuvre. Nos remerciements vont à l'endroit de l'UMR Eco \& Sol pour les différentes analyses effectuées et à monsieur Jean-Luc CHOTTE (Directeur de recherche) pour les échanges et discussions scientifiques.

\section{REFERENCES}

Amir S, Hafidi M, Merlina G, Revel JC. 2005. Sequential extraction of heavy metals during composting of sewage sludge. Chemosphere, 59(6): 801-810. https://doi.org/10.1016/j.chemosphere.20 04.11.016.

Azeez JO, Van Averbeke W. 2010. Nitrogen mineralization potential of three animal manures applied on a sandy clay loam soil. Bioresource Technology, 101(14): 5645-5651.

DOI: https://doi.org/10.1016/j.biortech.2010.0 1.119 .

Baharuddin AS, Wakisaka M, Shirai Y, Abd Aziz S, Abdul Rahman NA, Hassan MA. 2009. Co-composting of empty fruit bunches and partially treated palm oil mill effluents in pilot scale. Int. J. Agric. Res., 4(2): 69-78. DOI: 10.3923/ijar.2009.69.78.

Ben Ayed L, Hassen A, Jedidi N, Saidi N, Bouzaiane O, Murano F. 2005. Caractérisation des paramètres physicochimiques et microbiologiques au cours d'un cycle de compostage d'ordures ménagères. Déchets - revue francophone d'écologie industrielle, 40(4) : 11p.

Bessou C, Verwilghen A, Beaudoin-Ollivier L, Marichal R, Ollivier J, Baron V, Bonneau X, Carron M-P, Snoeck D, Naim M, Aryawan AAK, Raoul F, Giraudoux P, Surya E, Sihombing E, and Caliman J-P. 2017. Agroecological practices in oil palm plantations: examples from the field. OCL., 24(3). DOI:

https://doi.org/10.1051/ocl/2017024

Biaou ODB, Saidou A, Bachabi F-X, Padonou GE, Balogoun I. 2017. Effet de l'apport de différents types d'engrais organiques sur la fertilité du sol et la production de la carotte (Daucus carota L.) sur sol 
ferralitique au sud Bénin. Int. J. Biol. Chem. Sci., 11(5): 2315-2326. DOI: https://dx.doi.org/10.4314/ijbcs.v11i5.2

Biékré AHT, Tié BT, Dogbo DO. 2018. Caractéristiques physico-chimiques des composts à base de sous-produits de ferme de Songon en Côte d'Ivoire. Int. J. Biol. Chem. Sci., 12(1): 596-609. DOI: https://dx.doi.org/10.4314/ijbcs.v12i1.45

Brou YT, Chaléard J-C. 2007. Visions paysannes et changements environnementaux en Côte d'Ivoire. Ann. Géo., 653 : 65-87.

Charnay F. 2005. Compostage des déchets urbains dans les Pays en Développement : élaboration d'une démarche méthodologique pour une production pérenne de compost. Thèse de doctorat, Université de Limoges, $229 \mathrm{p}$.

Debril J, Matrat R, Haury J. 2005. Gestion des déchets de Jussie par compostage. INRA, Agrocampus Rennes, p. 37.

Elherradi E, Soudi B, Elkacemi K. 2003. Évaluation de la minéralisation de l'azote de deux sols amendés avec un compost d'ordures ménagères. Étude et Gestion des Sols, 10: 139-154.

Ettien DJB, Gnahoua JB, Kouadio KKH, Koné B, N'Zué B, Kouao AAF, De Neve S, Boeckx P. 2016. Soil fertility in land use for sustainable food crops production in the southern Côte d'Ivoire. Agriculture and Biology Journal of North America, 7(1): 19-26. DOI: 10.5251/abjna.2016.7.1.19.26

Francou C. 2003. Stabilisation de la matière organique au cours du compostage de déchets urbains : Influence de la nature des déchets et du procédé de compostage-Recherche d'indicateurs pertinents, Thèse de Doctorat, Institut national agronomique Paris-Grigon, 289 p.

GAB 44. 2015. Améliorer la fertilisation organique et la durabilité des exploitations bio. GAB 44, 23 p.

Hassen A, Belguith K, Jedidi N, Cherif A, Cherif M, Boudabous A. 2001. Microbial characterization during composting of municipal solid waste.
Bioresource Technology, 80(3): 217225.

DOI:

https://doi.org/10.1016/S0960-

8524(01)00065-7

Houot S, Cambier Ph, Benoit P, Deschamps M, Jaulin A, Lhoutellier C, Barriuso E. 2009. Effet d'apports de composts sur la disponibilité de micropolluants métalliques et organiques dans un sol cultivé. Étude et Gestion des Sols, 16: 255-274.

Huang GF, Wu QT, Wong JWC, Nagar BB. 2006. Transformation of organic matter during co-composting of pig manure with sawdust. Bioresource Technology, 97: $1834-1842 . \quad$ DOI: https://doi.org/10.1016/j.biortech.2005.0 8.024

Jacquemard J-C. 2012. Le Palmier à Huile. Editions Quae-CTA, Presses agronomiques de Gembloux : Gembloux.

Khalil MI, Hossain MB, Schmidhalter U. 2005. Carbon and nitrogen mineralization in different upland soils of the subtropics treated with organic materials. Soil Biology and Biochemistry, 37(8): 1507-1518. DOI: https://doi.org/10.1016/j.soilbio.2005.01. 014

Koledzi EK. 2011. Valorisation des déchets solides urbains dans les quartiers de Lomé (Togo): approche méthodologique pour une production durable de compost. Thèse de doctorat de l'Université de Lomé (TOGO) en cotutelle avec l'université de limoges, faculté des sciences et techniques, $199 \mathrm{p}$.

Koné B, Diatta S, Saïdou A, Akintayo I, Cissé B. 2009. Réponses des variétés interspécifiques du riz de plateau aux applications de phosphate en zone de forêt au Nigeria. Canadian Journal of Soil Science, 89(5): 555-565. DOI: 10.4141/CJSS08086

Lim KC, Zaharah AR. 2002. The effects oil palm empty fruit bunches on oil palm nutrition and yield, and soil chemical properties. Journal of Oil Palm Research, 14(2): 1-9. 
Masunga RH, Uzokwe VN, Mlay PD, Odeh I, Singh A, Buchan D, Neve SD. 2016. Nitrogen mineralization dynamics of different valuable organic amendments commonly used in agriculture. Applied Soil Ecology, 101: 185-193. https://doi.org/10.1016/j.apsoil.2016.01. 006

Mérigout P. 2006. Etude du métabolisme de la plante en réponse à l'apport de différents fertilisants et adjuvants culturaux. Influence des phytohormones sur le métabolisme azoté. Thèse de doctorat, Institut national agronomique ParisGrigon, $110 \mathrm{p}$.

Misra RV, Roy RN, Hiraoka H. 2005. Méthodes de compostage au niveau de l'exploitation agricole. Cahiers techniques de la FAO, documents de travail sur les terres et les eaux, $35 \mathrm{p}$.

Pakou C, Kornaros M, Stamatelatou K, Lyberatos G. 2009. On the fate of LAS, NPEOs and DEHP in municipal sewage sludge during composting. Bioresour. Technol., 100(4): 1634-1642. DOI: https://doi.org/10.1016/j.biortech.2008.0 9.025

Pansu M, Thuriès L, Larre-Larrouy MC, Bottner P. 2003. Predicting N transformations from organic inputs in soil in relation to incubation time and biochemical composition. Soil Biology \& Biochemistry, 35(3): 353-363. DOI : https://doi.org/10.1016/S00380717(02)00285-7

Record. 2002. Aide à la définition des déchets dits biodégradables, fermentescibles, méthanisables, compostables. Etude Record n 00-0118/1A, $151 \mathrm{p}$.

Salètes S, Fiorelli JL, Vuichard N, Cmabou J, Olesen JE, Hacala S, Sutton MA, Fuhrer J, Soussana JF. 2004. Proceedings of the International Conference "Greenhouse gas emissions from agriculture -
Mitigations options and strategies". Leipzig, 10-12th February 2004: 203208.

Schuchardt F, Darnoko D, Guritno P. 2002. Composting of empty oil palm fruit bunch (EFB) with simultaneous evaporation of oil mill waste water (POME). International oil palm conference, Nusa Dua, Bali.

Singh R, Ibrahim MH, Esa N, Iliyana M. 2010. Composting of waste from palm oil mill: A sustainable waste management practice. Reviews in Environmental Science and Biotechnology, 9(4): 331-344. DOI: 10.1007/s11157-010-9199-2

Toundou O. 2016. Evaluation des caractéristiques chimiques et agronomiques de cinq composts de déchets et étude de leurs effets sur les propriétés chimiques du sol, la physiologie et le rendement du maïs (Zea mays L. Var. Ikenne) et de la tomate (Lycopersicum esculentum L. Var.) sous deux régimes hydriques Tropimech au Togo. Thèse de doctorat de l'université de Lomé en cotutelle avec l'université de Limoges, $213 \mathrm{p}$.

Weill A, Duval J. 2009. Les amendements organiques: fumiers et composts. Equiterre, $19 \mathrm{p}$.

Yoni M, Hien V, Abbadie L, Serpentié G. 2005. Dynamique de la matière organique du sol dans les savanes soudaniennes du Burkina Faso. Cahiers d'Agriculture, 14(6): 525-532.

Zorpas AA, Arapoglou D, Karlis P. 2003. Waste paper and clinoptilolite as a bulking material with dewater anaerobically stabilized primary sewage sludge (DASPSS) for compost production. Waste Management, 23(1): 27-35. DOI: $10.1016 / \mathrm{S} 0956-$ 053X(02)00042-9. 